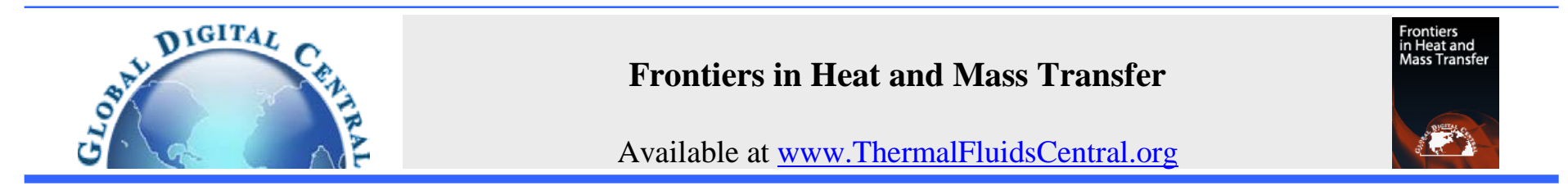

\title{
STUDY ON HEAT AND MASS TRANSFER AND NONLINEAR CHARACTERISTICS WITH THERMAL AND SOLUTAL SOURCE IN A CAVITY
}

\author{
Yubing $\mathrm{Li}^{\mathrm{a}}$, Mo Yang ${ }^{\mathrm{a}}$, Jian Li ${ }^{\mathrm{b}}$, Zhiyun Wang \\ a School of Energy and Power Engineering, University of Shanghai for Science and Technology, Shanghai 200093, China \\ ${ }^{b}$ Shanghai Aircraft Manufacturing Co., Ltd., Commercial Aircraft Corporation of China, Ltd., Shanghai 200436, China
}

\begin{abstract}
Thermalsolutal convection induced by mass and heat source in horizontal cavity is investigated numerically based on SIMPLE algorithm with QUICK scheme. The high-concentration heat source is placed in the square cavity, and the cavity wall is low temperature and low concentration. The smoke is used as the diffusion medium, and the flow field, temperature and concentration of the fluid under different Rayleigh number, buoyancy ratio $N c$, Soret numbers and Dufour numbers are analyzed systematically. Parameter study demonstrates that heat and mass transfer of thermosolutal convection are enhanced with increasing Rayleigh number or buoyancy ratio, and the couple-diffusive interaction has a significant linear effect on heat transfer and mass diffusion. The results also show that double-diffusive convection evolves from steady state, periodic oscillatory, double-period oscillation to chaos. Critical Rayleigh number of period oscillations and chaos declined with increasing buoyance ratio.
\end{abstract}

Keywords: mass and heat source, numerical simulation, heat and mass transfer, oscillation, chaos

\section{INTRODUCTION}

Double-diffusive buoyancy convection driven by thermal and solutal source could generate heat and mass transfer phenomena. For example, during the process of fire propagation, the fire source is not only the heat source, but also the mass source to generate pollutants. In addition, the combustion inside the boiler furnace also involves fluid flow induced by heat and mass source. During the double-diffusive convection process, thermal and solutal buoyancy and couple-diffusive interaction will produce many intriguing and complex nonlinear flow, which is also playing a key role in many engineering applications.

In the past, people focused on the natural convection with internal heat source, but there are few reports on the coupled heat and mass transfer flow with mass heat source. Tasaka (2011) studied the impact of a heat source distribution on natural convection induced by internal heating obtained by using simplified models of the distribution. Sankar (2005) examined maximum heat transfer under varying Rayleigh and Darcy numbers for different heat source lengths and locations in a vertical annulus filled with a fluid-saturated porous medium. A 2-D numerical study is performed by Karimi (2015) to observe natural convection from two heated horizontal cylinders confined in a square enclosure with isothermal walls at the heat sink temperature. It is noted that steady state flow and heat transfer undergoes periodical oscillation, and ultimately chaotic oscillation in special positions of cylinders. Double-diffusion convection with a mass heat source is inspired by natural convection with an internal heat source. Double-diffusion convection with a mass heat source is inspired by natural convection with an internal heat source. Effect of Stefan number and geometry of the heaters on the melting process are also investigated (Li et al., 2013) in a discretely heated rectangular enclosure obtained using an interfacial tracking method. Lin (2000) originally studied the natural convection phenomenon of coaxial high-temperature line sources in cylinders. Juncu
(2005) expanded on Lin's model by adding the mass source and analyzed the influence of the conductivity ratio on the local average Nu numbers at different values of the wire diameter. Hill (2005) used linear and nonlinear stability theory to analyze the double-diffusion convection in a fluid-saturated porous layer with a concentration of internal heat source. Nazari (2015) simulated the double-diffusion of closed-cavity endogenous heat source by LBM method, and analyzed the heat and mass transfer of different buoyancy ratio $\mathrm{N}(-4<N<0)$ and different mass source geometries. Nonlinear stability analysis of double-diffusive convection in a horizontal fluid saturated porous layer has been investigated (Deepika et al., 2016). Matta (2017) studied the thermal swell instability of oblique fluid double-diffusion convection in saturated porous layers. Xu (2016) studied the heat and mass transfer convection of a mass-containing heat source in a cavity with different exit locations.

As for thermosolutal convection induced by temperature and concentration difference, it is very necessary to build the doublediffusive interaction, since more studies of the nonlinear has widely reported that Soret and Dufour effects plays an important role in heat and mass transfer (Weaver et al., 1991). The Platten (2014) conducted experiment to compare the different techniques for measuring the Soret coefficient and obtains the standard value of the liquid Soret coefficient. Malashetty and Gaikwad (2007) studied the effects of the Soret coefficient and the Dufour coefficient on the double-diffusion convection of an unbounded vertical layered system with horizontal temperature and concentration gradients. Gaikwad (2014) considered the effects of coupled stress and increasing the internal heat source in the Gaikwad model to study the effects of Soret and Dufour numbers and Prandtl numbers on stationary and oscillatory convection. Altawallbeh et al. (2013) analyzed the effects of internal heat source and Soret effect on the double-diffusion convection in porous media. Wang et al. (2014, 2015 \& 2016) used the SIMPLE algorithm of QUICK format to simulate the temperature and concentration coupled heat and mass transfer law, and

*Corresponding author. Email: yangm@usst.edu.cn 
obtained the change of fluid flow in the cavity with the different dimensionless parameters. Nithyadevi and Yang (2009) used water as the medium to simulate the double-diffusion flow in the closed cavity by SIMPLE algorithm, and obtained the effects of different boundary conditions, Rayleigh number, Dufour number and Soret number on heat and mass transfer. Bhuvaneswari (2011) investigated the heat transfer and mass transfer models of mixed convection in a double-sided coverdriven cavity considering the Soret effect. However, fewer investigations pointed out that the thermal and solutual source is of great significant in the nonlinear routes of the heat and mass transfer. Xu (2019) used the LBM method to study the effects of different Soret numbers and Dufour numbers on convection and heat and mass transfer heat transfer in a cavity with a heat source. The results show that when $L e=2, \operatorname{Pr}=1$ and $R a=5 \times 10^{6}$, the fluid stability increases with the increase of $S_{r}$ and $D_{f}$.

In summary, few results have been reported in the heat and mass transfer model containing the mass heat source, and most of them only based on the air and water, and there are fewer articles that use smoke as the medium and consider the nonlinearity of coupling heat and mass transfer with the mass heat source. Therefore, this paper will explore the smoke convective heat and mass transfer and nonlinear characteristics of solutal and thermal source using numerical simulation. The influence of different parameters on the heat and mass transfer characteristics is analyzed according to the numerical results.

\section{PROBLEM STATEMENT AND FORMULATION}

Figure 1 shows physical model for a double-diffusion natural convection symmetric square cavity with heat and mass source. The aspect ratio is $1: 2$, and the length of inner square source is one quarter to height of cavity. Uniform temperatures and concentrations are specified at the outer four surfaces $\left(T_{l}, C_{l}\right)$ and inner $\left(T_{h}, C_{h}\right)$, where $T_{h}>T_{l}, C_{h}>C_{l}$, and the high temperature and high concentration square source coincides in the middle between center point and left wall and right wall of cavity. Initially, cavity is filled with a binary medium of air and smoke $\left(T_{0}, C_{0}\right)$. Due to the temperature and concentration difference between two squares, density gradient occurs and results in the thermosolutal buoyancy convection and diffusion.

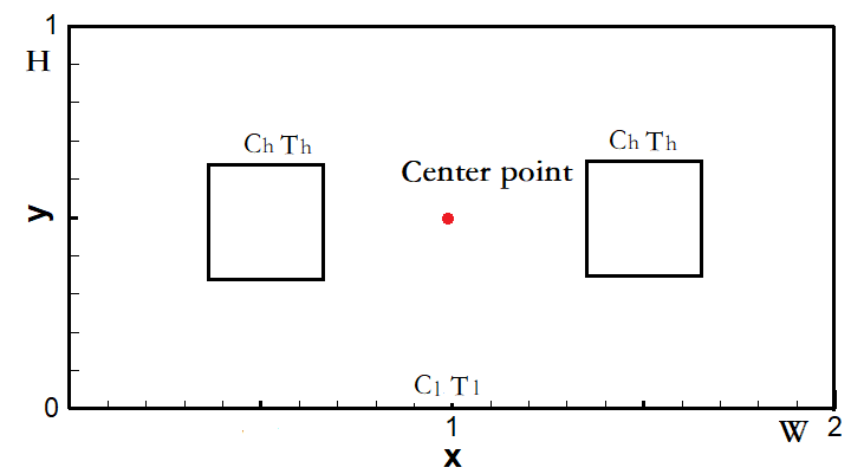

Fig. 1 Physical model and coordinate system

It is assumed that fluid in the cavity is a Newtonian fluid, and there is no chemical reaction or thermal radiation. According to the Boussinesq hypothesis the density in the gravity term in the momentum equation varies with the concentration temperature, and other properties in the other governing equations are constant and independent of time and temperature. The wall is non-slip boundary conditions.

Based on the above assumptions, the heat and mass transfer dimensionless equations inside the square cavity with Soret and Dufour effects is

$$
\frac{\partial U}{\partial X}+\frac{\partial V}{\partial Y}=0
$$

$$
\begin{aligned}
& \frac{\partial U}{\partial \tau}+U \frac{\partial U}{\partial X}+V \frac{\partial U}{\partial Y}=-\frac{\partial P}{\partial X}+\operatorname{Pr}\left(\frac{\partial^{2} U}{\partial X^{2}}+\frac{\partial^{2} U}{\partial Y^{2}}\right) \\
& \frac{\partial V}{\partial \tau}+U \frac{\partial V}{\partial X}+V \frac{\partial V}{\partial Y}=-\frac{\partial P}{\partial Y}+\operatorname{Pr}\left(\frac{\partial^{2} V}{\partial X^{2}}+\frac{\partial^{2} V}{\partial Y^{2}}\right)+\operatorname{Ra} \operatorname{Pr}(\theta+N C) \\
& \frac{\partial \theta}{\partial \tau}+U \frac{\partial \theta}{\partial X}+V \frac{\partial \theta}{\partial Y}=\left(\frac{\partial^{2} \theta}{\partial X^{2}}+\frac{\partial^{2} \theta}{\partial Y^{2}}\right)+D_{f}\left(\frac{\partial^{2} C}{\partial X^{2}}+\frac{\partial^{2} C}{\partial Y^{2}}\right) \\
& \frac{\partial C}{\partial \tau}+U \frac{\partial C}{\partial X}+V \frac{\partial C}{\partial Y}=\frac{1}{L e}\left(\frac{\partial^{2} C}{\partial X^{2}}+\frac{\partial^{2} C}{\partial Y^{2}}\right)+S_{r}\left(\frac{\partial^{2} \theta}{\partial X^{2}}+\frac{\partial^{2} \theta}{\partial Y^{2}}\right)
\end{aligned}
$$
follows:

Where dimensionless parameters in the equation are defined as

$$
\begin{aligned}
& (U, V)=\frac{(u, v) W}{\alpha},(X, Y)=\frac{(x, y)}{W}, \theta=\frac{T-T_{l}}{T_{h}-T_{l}}, C=\frac{C-C_{l}}{C_{h}-C_{l}}, \tau=\frac{\alpha t}{W^{2}}, \\
& P=\frac{W^{2}\left(p+\rho_{0} g y\right)}{\rho_{0} \alpha^{2}}, \operatorname{Pr}=\frac{v}{\alpha}, L e=\frac{\alpha}{D}, R a=\frac{g W \beta_{T}\left(T_{h}-T\right)}{v \alpha}, \\
& N C=\frac{\beta_{C}\left(C_{h}-C_{l}\right)}{\beta_{T}\left(T_{h}-T_{l}\right)}, D_{f}=\frac{\kappa_{T C}\left(C_{h}-C_{l}\right)}{\alpha\left(T_{h}-T_{l}\right)}, S_{r}=\frac{\kappa_{C T}\left(T_{h}-T_{l}\right)}{D\left(C_{h}-C_{l}\right)}
\end{aligned}
$$

Among them, $v$ is the kinematic viscosity, $\alpha$ is the thermal diffusion coefficient, $D$ is the mass diffusion coefficient, and $g$ is the gravitational acceleration. $\kappa_{T C}$ and $\kappa_{C T}$ are Dufour and Soret coefficients.

The boundary conditions for (1) (5) are defined as:

$$
\begin{aligned}
& X=0 \text { or } X=W, U=V=0, \theta=0, C=0 \\
& Y=0 \text { or } Y=H, U=V=0, \theta=0, C=0
\end{aligned}
$$

The boundary conditions of heat and mass source are

$$
\begin{aligned}
& \frac{3}{16} W<X<\frac{5}{16} W, \frac{9}{16} W<X<\frac{13}{16} W, U=V=0, \theta=1, C=1 \\
& \frac{3}{8} H<Y<\frac{5}{8} H, U=V=0, \theta=1, C=1
\end{aligned}
$$

Initial conditions:

$$
\tau=0, U=V=0, \theta=0, C=0
$$

Local Nusselt and Sherwood number with Soret and Dufour effect can be defined as below:

$$
\begin{aligned}
& N u=\frac{\partial \theta}{\partial Y}+D_{\mathrm{f}} \frac{\partial C}{\partial Y} \\
& S h=\frac{\partial C}{\partial Y}+S_{r} \frac{\partial \theta}{\partial Y}
\end{aligned}
$$

The average $\overline{N u}$ and $\overline{S h}$ are:

$$
\begin{aligned}
& \overline{N u}=\int_{0}^{1} N u(X) d X \\
& \overline{S h}=\int_{0}^{1} S h(X) d X
\end{aligned}
$$

\section{NUMERICAL METHOD}

In this paper, the unsteady model of Soret and Dufour coupling effect is used. The finite volume method is used to discretize the controlling equation. The interface interpolation uses the third-order precision QUICK scheme, and the SIMPLE algorithm is used to deal with the 
velocity and pressure coupling. To ensure that the calculation results can converge, a sub-relaxation method is used in the iterative process. The convergence criterion for each time step is less than $1 \times 10^{-6}$.

\section{RESULTS AND DISCUSSION}

\subsection{Numerical method and assessment}

The grid-independence of numerical results is studied at a value Rayleigh number of $5 \times 10^{4}$ and $1 \times 10^{5}$. Here, the dimensionless parameter $D_{f}, S_{r}$ and $N c$ are zero, and $L e$ and $P r$ are 1.641 and 0.71.Table 1 shows five mesh sizes to complete the validity of grid time step $\Delta t=0.01$.The average Nusselt numbers shown in table 1 increase slightly and then remain unchanged as the total number of grid point changes from $100 \times$ 50 to $200 \times 100$. The relative error is calculated in table 1 , which is defined as ratio of $\mid N u$ (different grids)- $N u(160 \times 80) \mid$ and $N u(160 \times 80)$. When grid numbers reach to $160 \times 80$, the relative error of Nusselt number is less than $0.1 \%$. So the grid numbers of $160 \times 80$ will be used.

Table 1 Average Nusselt number under different grid numbers

\begin{tabular}{|l|l|l|l|l|}
\hline $\mathrm{Nu}$ & $R a=5 \times 10^{4}$ & $R a=1 \times 10^{5}$ & $\mathrm{err}_{1}$ & $\mathrm{err}_{2}$ \\
\hline $100 \times 50$ & 1.9333 & 2.2014 & $5.63 \%$ & $9.6 \%$ \\
\hline $120 \times 60$ & 2.0458 & 2.4267 & $0.14 \%$ & $0.41 \%$ \\
\hline $160 \times 80$ & 2.0487 & 2.4369 & 0 & 0 \\
\hline $180 \times 90$ & 2.0497 & 2.4379 & $0.048 \%$ & $0.041 \%$ \\
\hline $200 \times 100$ & 2.0493 & 2.4379 & $0.029 \%$ & $0.041 \%$ \\
\hline
\end{tabular}

In addition, the validation of time step is performed with the grid number of $160 \times 80$. Table 2 shows the relationship between time step and average Nusselt number under different Rayleigh number. It can be observed that, the relative error is lower than $0.01 \%$ for several time steps below 0.01 , and the relative error is increasing for time steps above 0.01 . So time step is taken as 0.01 in this paper reaching good compromise between CPU and precision.

Table 2 Average Nusselt number under different time step

\begin{tabular}{|l|l|l|l|l|}
\hline $\mathrm{Nu}$ & $\mathrm{Ra}=5 \times 10^{4}$ & $R a=1 \times 10^{5}$ & err $_{3}$ & err $_{4}$ \\
\hline 0.001 & 2.0491 & 2.436 & $0.01 \%$ & $0.009 \%$ \\
\hline 0.005 & 2.0491 & 2.436 & $0.007 \%$ & $0.005 \%$ \\
\hline 0.01 & 2.0491 & 2.436 & 0 & 0 \\
\hline 0.05 & 2.4380 & 2.4379 & $19 \%$ & $0.08 \%$ \\
\hline
\end{tabular}

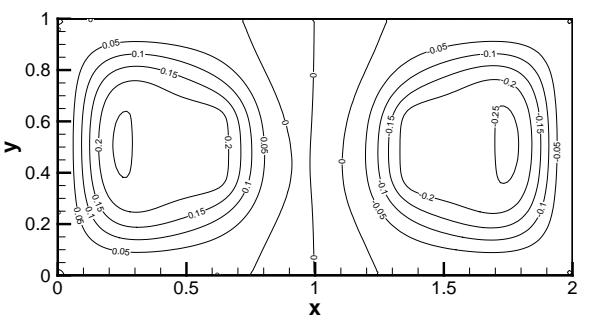

(a)

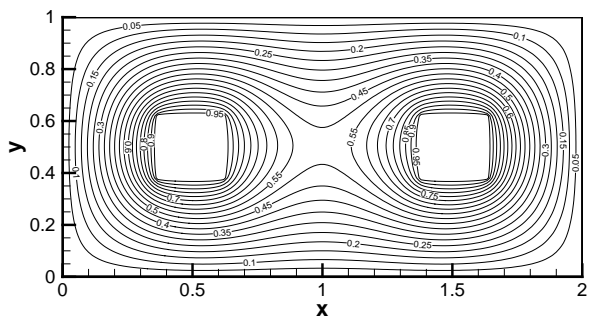

(b)

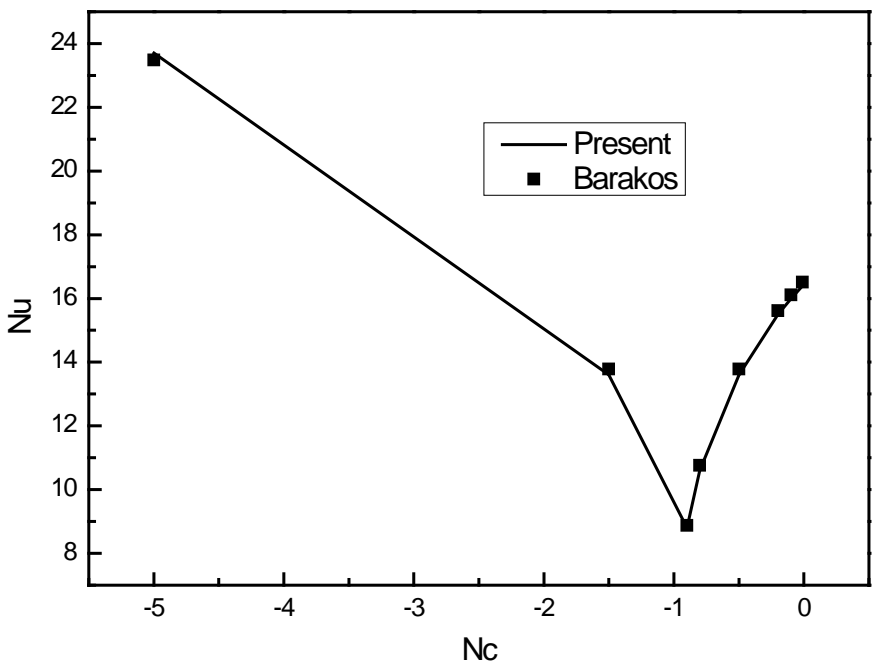

Fig. 2 The comparison result for average Nusselt number under numerical results

The dimensionless parameter $L e=1, \operatorname{Pr}=0.71$, when there is no Soret and Dufour effect, ie $D_{f}=S_{r}=0$, the initial concentration in each square cavity is 0 . Similar to the Beghein (1992) model, the buoyancy Nc ranges from -0.01 to -5 , and the experimental results obtained are in good agreement with the simulation results (shown in Fig. 2).

\subsection{Effect of Rayleigh number}

Figure 3 (a) 3 (c) and Figure 4 (a) 4 (c) presents steady-state solution of streamline, temperature and concentration for $R a=5 \times 10^{2}$ and $R a=$ $5 \times 10^{3}$, respectively. It is found that there exists two-vortex conductiondominated flow pattern at Rayleigh number below $10^{3}$, and four vortices induced by convection-dominated flow for Rayleigh number above $5 \times$ $10^{3}$. Contours of temperature and concentration distributions are concentric circles at a Rayleigh number of $5 \times 10^{2}$. Also, contours near sources are denser, but contours in the middle of cavity are thin due to the zero velocity according to the streamline. As $R a$ increases to $5 \times 10^{3}$, heat and mass transfer are enhanced. Temperature on the top of the source is larger than the other sides because double diffusion convection driven by the upward thermal and solute buoyancy. So the small vortex on the left is counterclockwise with the corresponding one on the left clockwise. The concentration distribution is similar to the temperature.

Fig. 3 Steady solution for $R a=5 \times 10^{2}$, (a) streamline, (b) isotherm, (c) isosolute

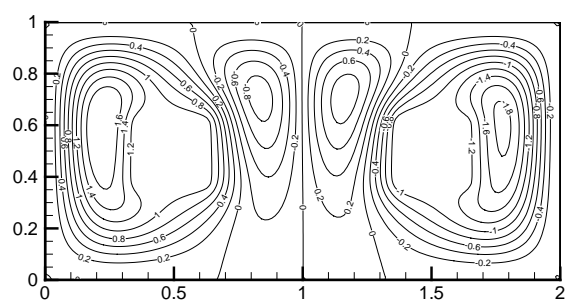

(a)

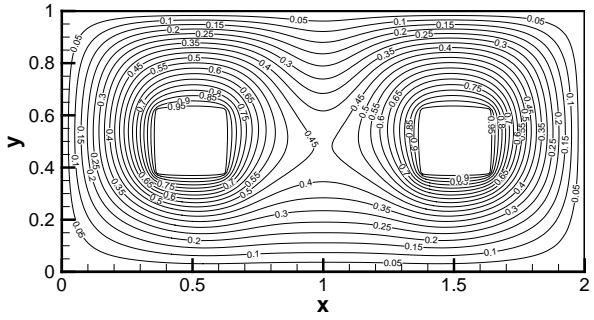

(b)

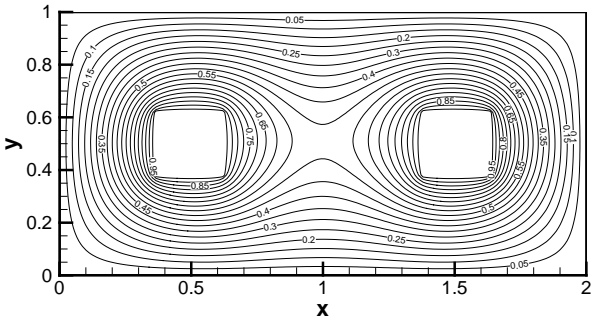

(c)

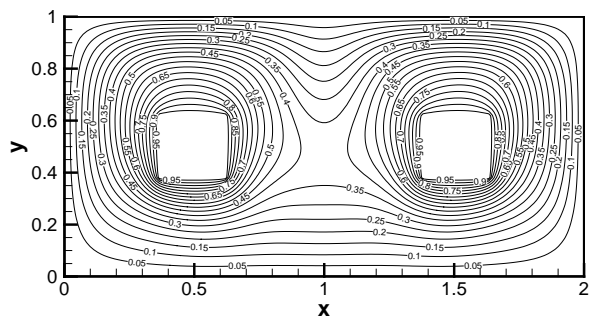

(c)

Fig. 4 Steady solution for $R a=5 \times 10^{3}$, (a) streamline, (b) isotherm, (c) isosolute 


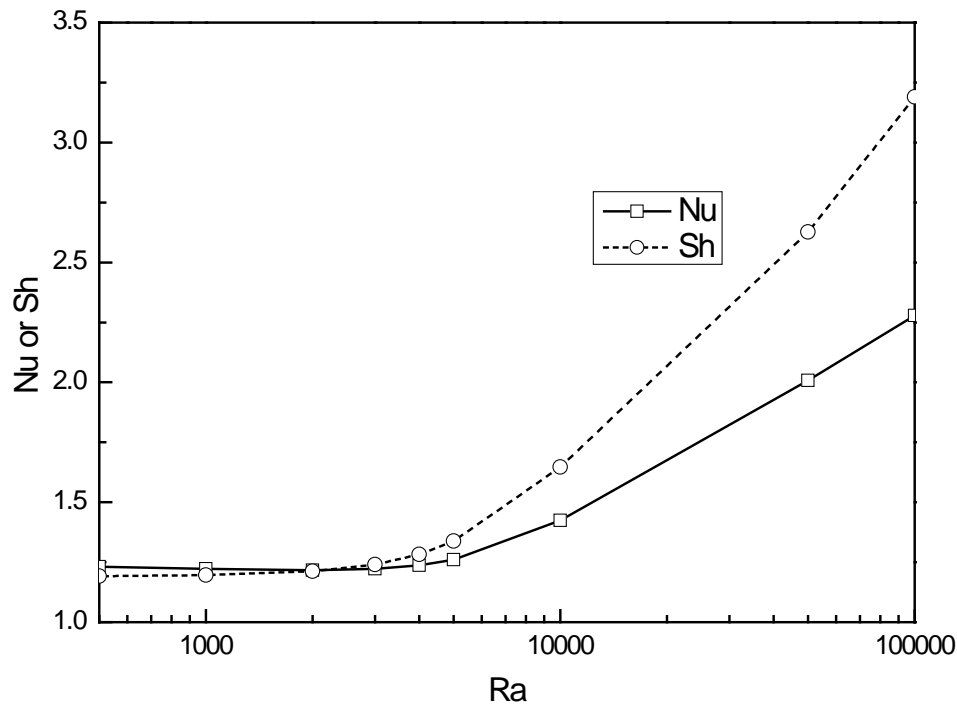

Fig. 5. Average Nusselt and Sherwood number

Figure 5 presents the average Nusselt and Sherwood number for various $R a$. It is observed that $S h$ remain constant and then increase. Since conduction-dominated flow evolves into convection-dominated. However, Nu decreases slightly for case of $2 \times 10^{3} \leqslant R a<4 \times 10^{3}$ and then bump up for Ra above $4 \times 10^{3}$. The reason is that system is converted from two symmetric eddies (Fig. 3 (a)) to four (Fig. 4 (a)) symmetric vortices orderly, and the heat transfer is weak at the beginning of the development of the four vortices. Also, the heat transfer is easily affected by the vortex structure, but mass transfer is not affected by the vortex structure. The average $\mathrm{Nu}$ is smaller than $\mathrm{Sh}$ because mass transfer is stronger near the high temperature and concentration source $(L e=1.641$ $<1)$.

\subsection{Effect of buoyancy ratio}

Figure 6 shows the streamlines, isothermal and isosolutal distribution when $R a=10^{4}$ and $N c=-2$. Comparing with the buoyance ratio $N c=1$ as shown in Fig. 4 (a). Fig. 6 illustrates that the heat and mass in the center of cavity of the upper wall sink, since double-diffusive convection is induced by downward resultant force and thermal and solutal buoyancies in the opposite direction. Therefore, the left one of two vortices between two sources is counter-clockwise. And stagnant zone near the upper wall is obtained as depicted by Fig. 6 (a). Meanwhile, it can be seen from the Fig. 6 (b) and Fig. 6 (c) that isotherm and isoconcentration on the bottom of source are denser, and counters will become more concentrated on with decreasing buoyance ratio Nc.

Figure 7 shows effect of buoyance ratio on the thermalsolutal convection in the range $-7.5 \leqslant N_{c} \leqslant 7.5$ at a Rayleigh number of $5 \times$ $10^{3}$. It can be seen that Nusselt and Sherwood numbers both are decreasing and then increasing with increasing Nc. The minimum mass transfer rate appears at $N c=-1$, because the solutal buoyance is equal to thermal buoyance but in opposite directions and the fluid is driven by conduction-dominated at this point. However, the minimum heat transfer rate is at $N_{C}=0$ instead of $N_{C}=-1$, because four asymmetric steady vortex patterns more irregular than two symmetry vortices as shown in the illustration. And mass transfer is not affected by the flow pattern. Mass transfer is larger than heat with positive buoyance ratio. Lines of Nusselt and Sherwood numbers are parallel each other with negative buoyance ratio. Since solutal buoyancy keeps up rising for $N c$ from -1 to -7.5 , the convection remains still conduction. Double diffusion is changing consistently.

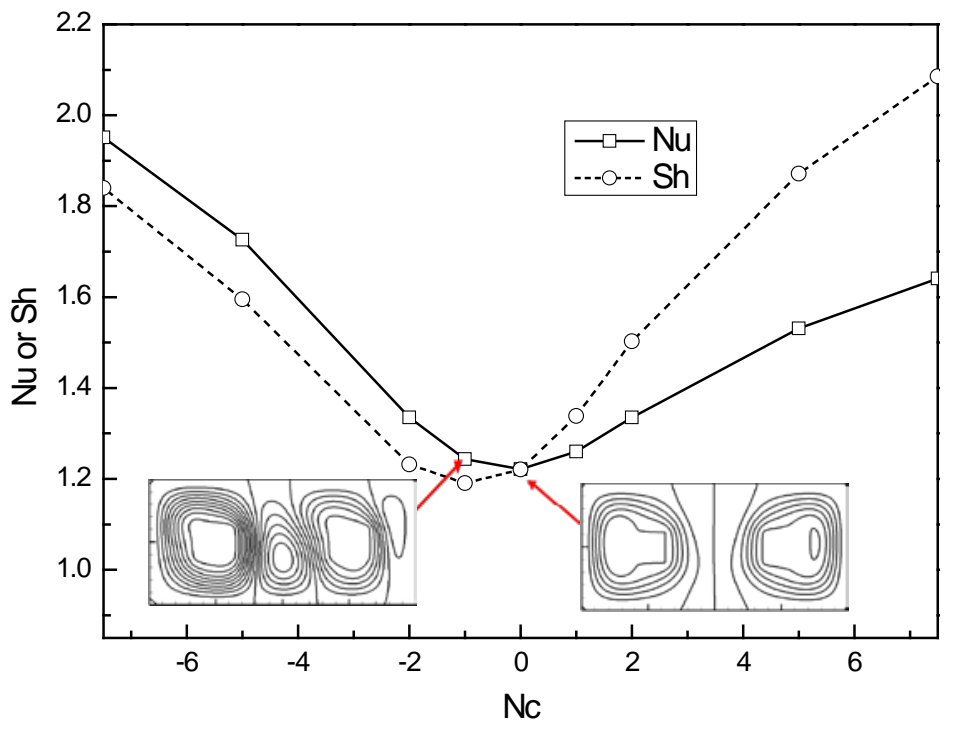

Fig. 7 Average Nusselt and Sherwood number

\subsection{Effect of Soret and Dufour number}

Figure 8 shows the Soret and Dufour effect on the thermalsolutal convection. It can be noted average Nusselt and Sherwood number grow congruously with Soret as shown in Fig. 8 (a), at a Dufour number of 0.1. Although weaken Dufour effect on heat and mass transfer is constant, Soret effect become more intense with increasing Soret number. That is because the effect of thermal buoyancy on thermalsolutal convection rises up. And the Dufour effect on the thermalsolutal convection is similar with the Soret effect on that. It should be known that there is no significant difference in the streamline, isotherm and isosolute. Consequently, the growth rate of Nusselt and Sherwood number are calculated. As Soret effect become intense, heat transfer rate is increased by $21 \%$ and mass transfer $35 \%$ in Fig. 8 (a). However, the slope of the Nusselt is steeper in the Fig. 8 (b). As Dufour effect become intense, heat transfer rate is increased by $25 \%$ and mass transfer $34 \%$.Therefore, both Soret numbers due to the temperature gradient and Dufour number due to the concentration gradient enhance the heat and mass transfer. But concentration difference and temperature difference have great impact on mass transfer. Also, mass diffusion is more exacerbated at temperature difference and concentration difference than thermal convection.

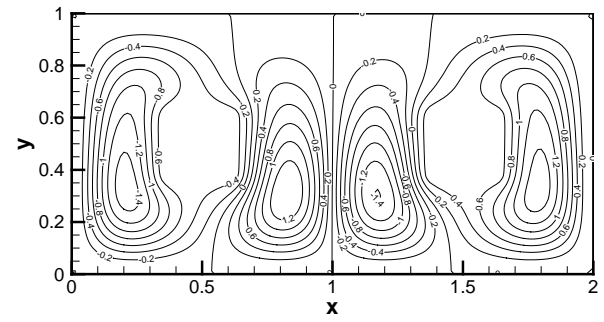

(a)

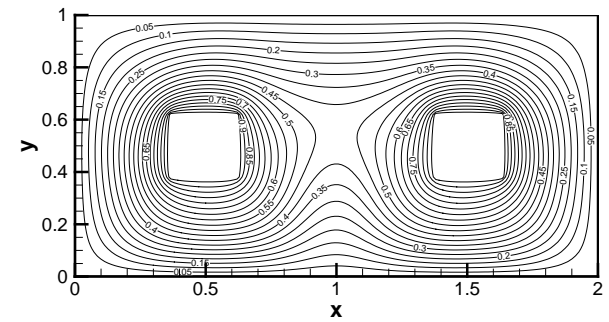

(b)

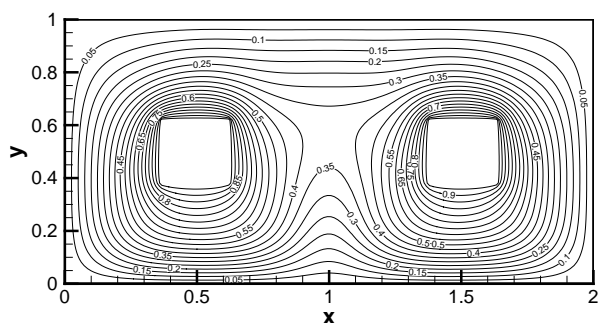

(c)

Fig. 6 Steady solution for $N c=-2$, (a) streamline, (b) isotherm, (c) isosolute 


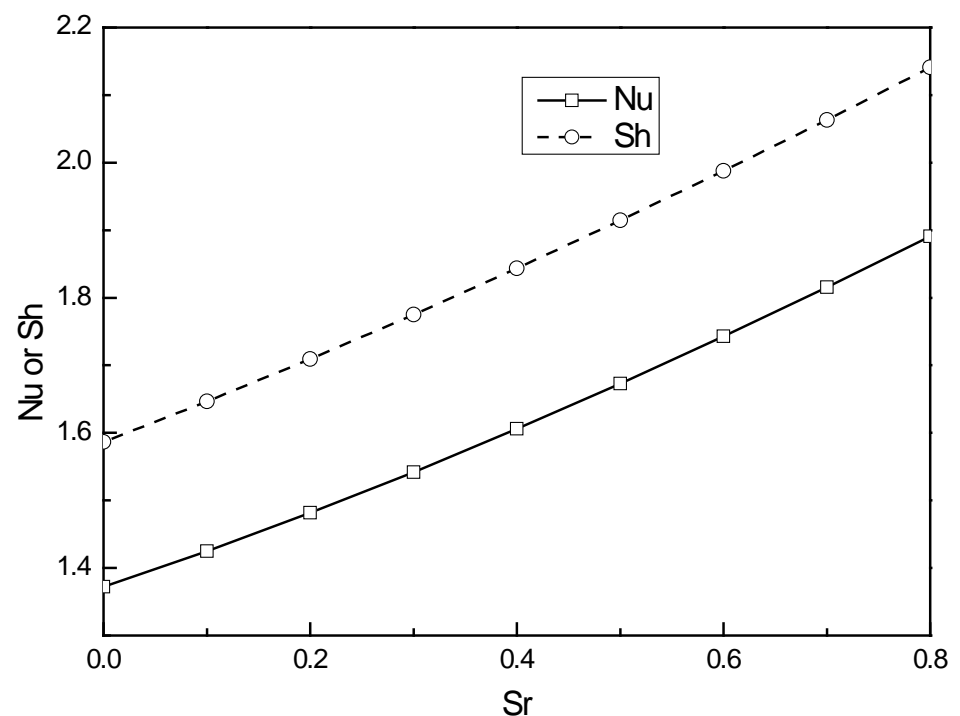

(a) $D f=0.1$

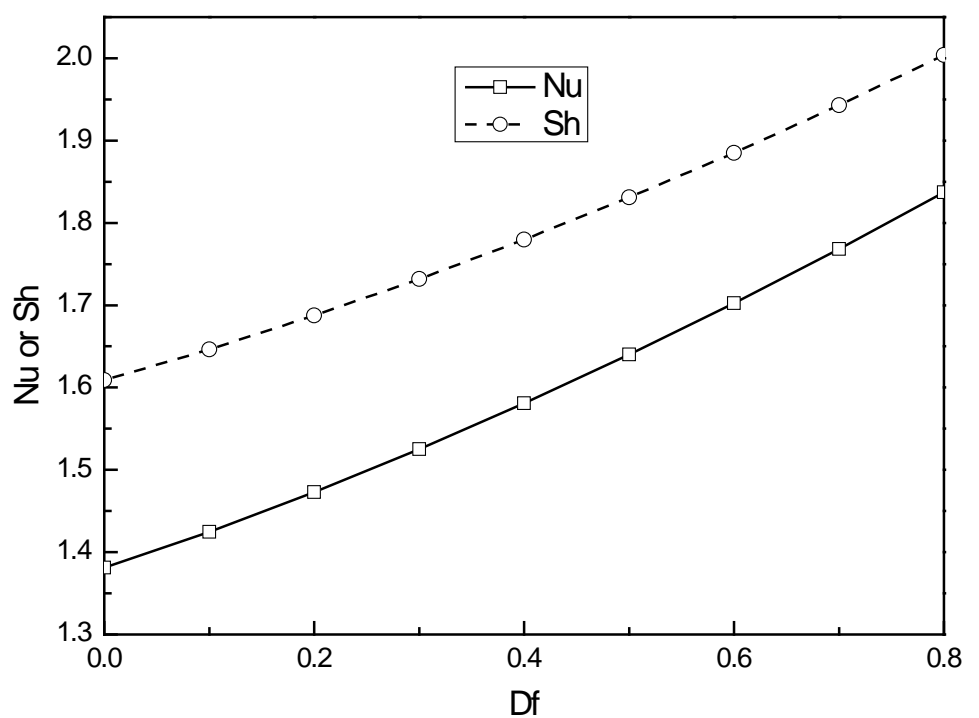

(b) $\mathrm{Sr}=0.1$

Fig. 8 Average Nusselt and Sherwood number
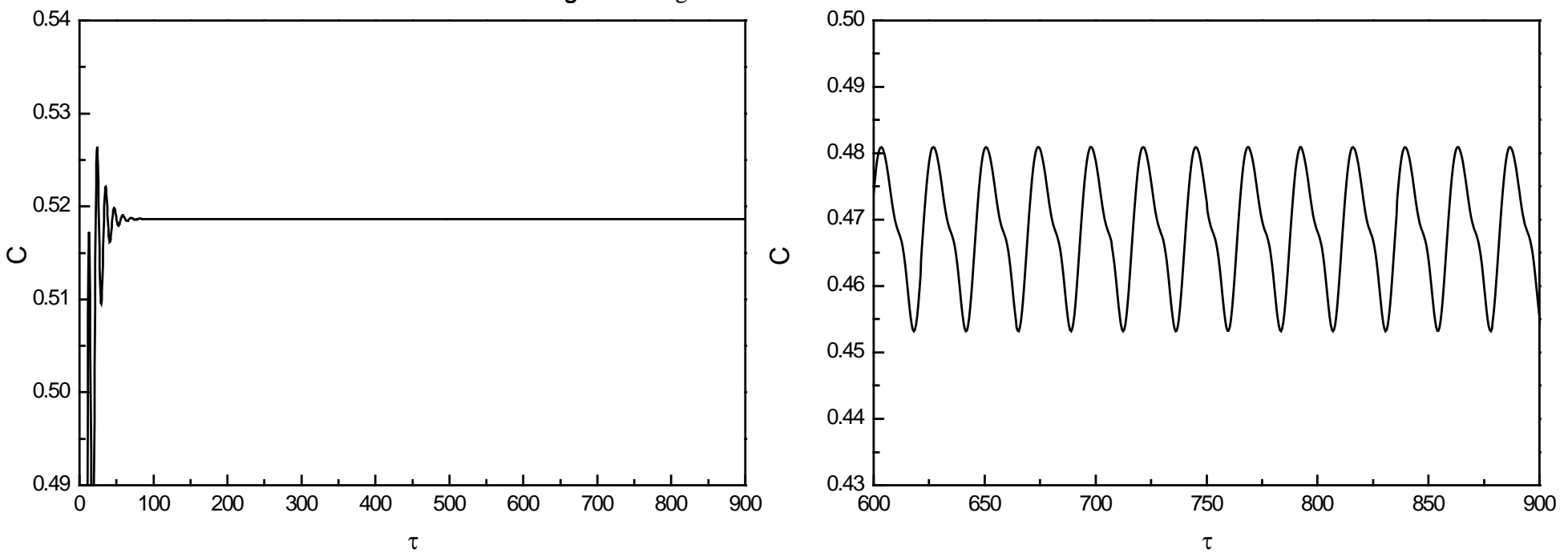

(a) $\operatorname{Rz} 2 \times 10^{4}$

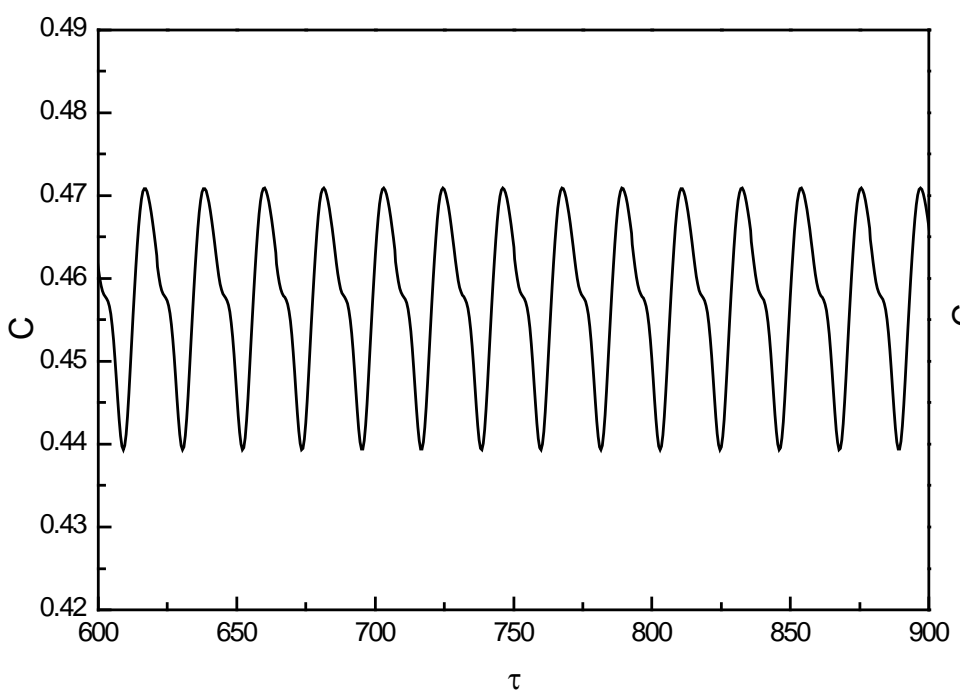

(c) $\operatorname{Ra} 5 \times 10^{4}$

(b) $\operatorname{Rz} 4 \times 10^{4}$

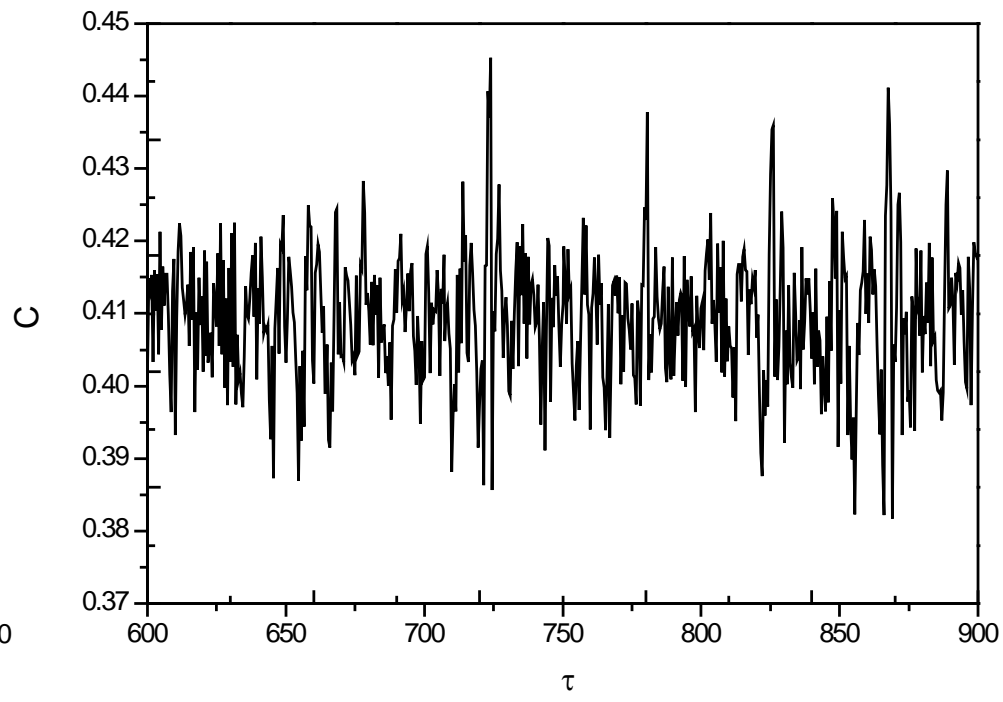

(d) $\operatorname{Ra} 9 \times 10^{5}$

Fig. 9 Time history of concentration for (a) $R a=2 \times 10^{4}$, (b) $R a=4 \times 10^{4}$, (c) $R a=5 \times 10^{4}$, (d) $R a=9 \times 10^{5}$ 


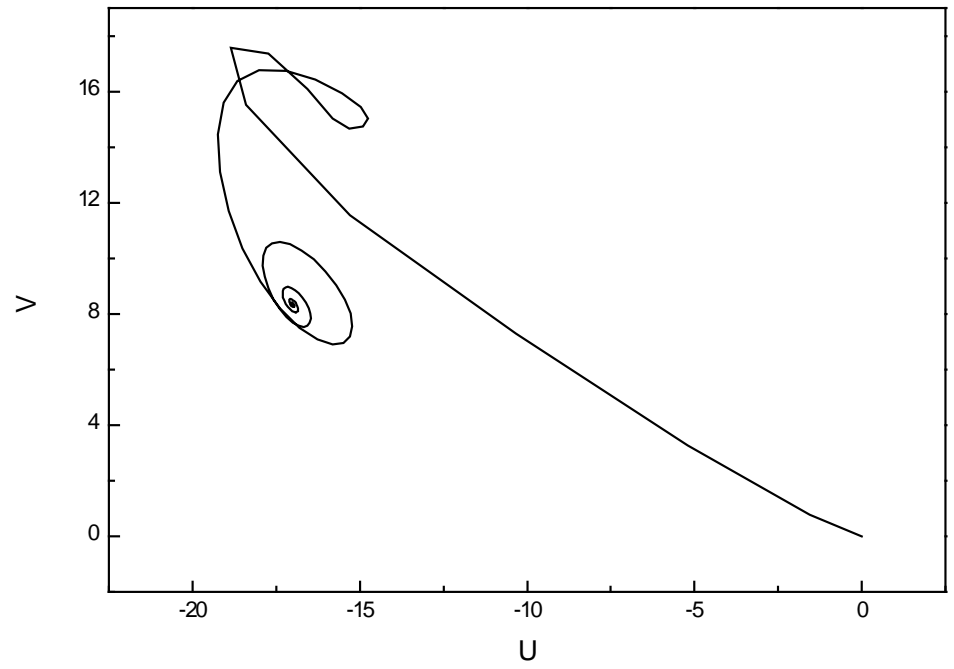

(a) $\mathrm{Ra}=2 \times 10^{4}$

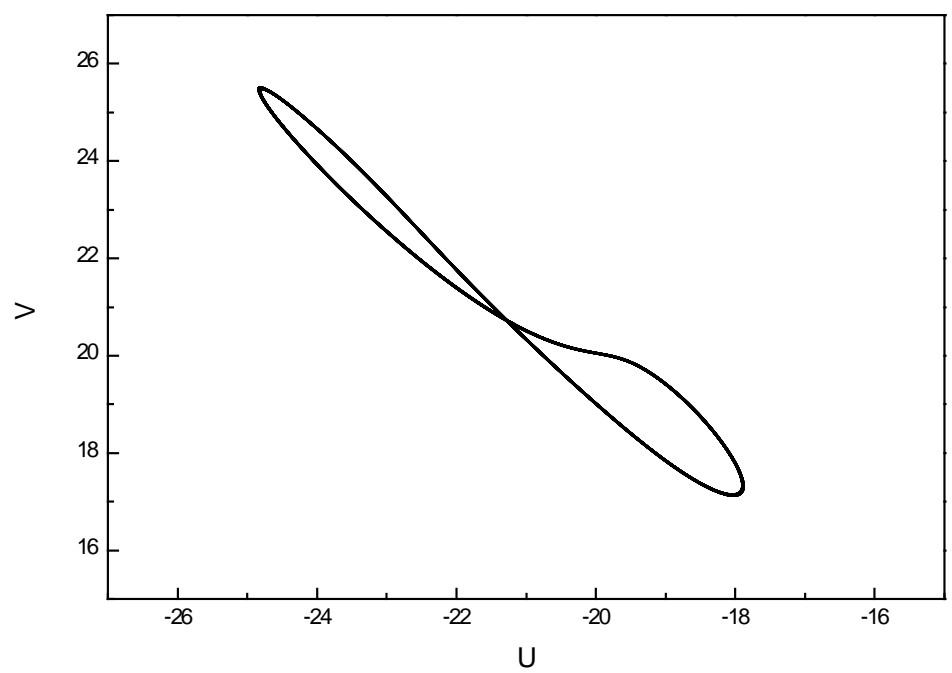

(c) $\operatorname{Ra}=5 \times 10^{4}$

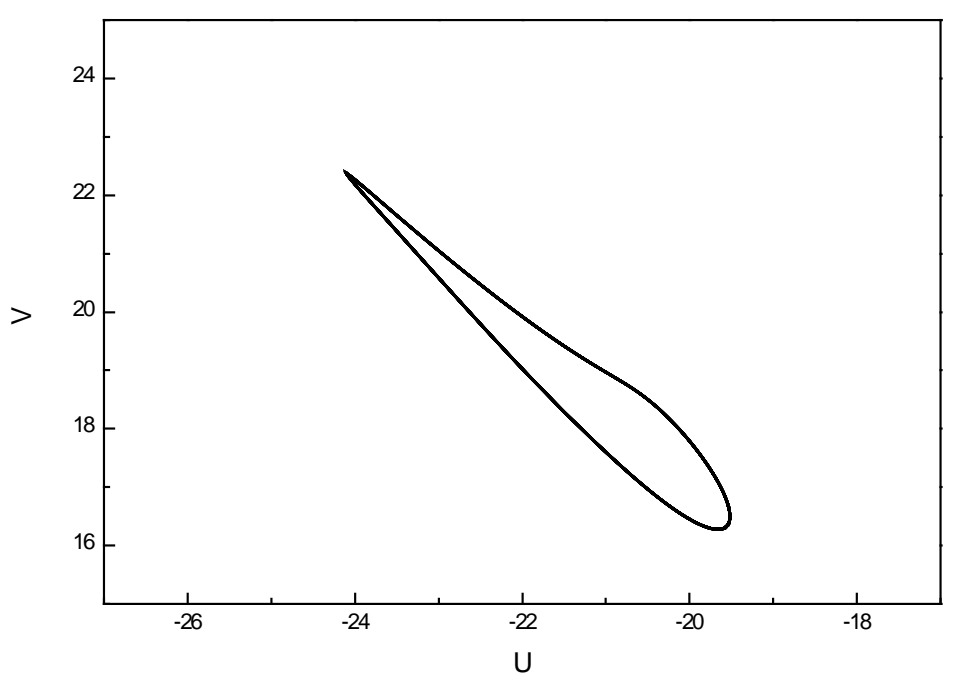

(b) $\mathrm{Ra}=4 \times 10^{4}$

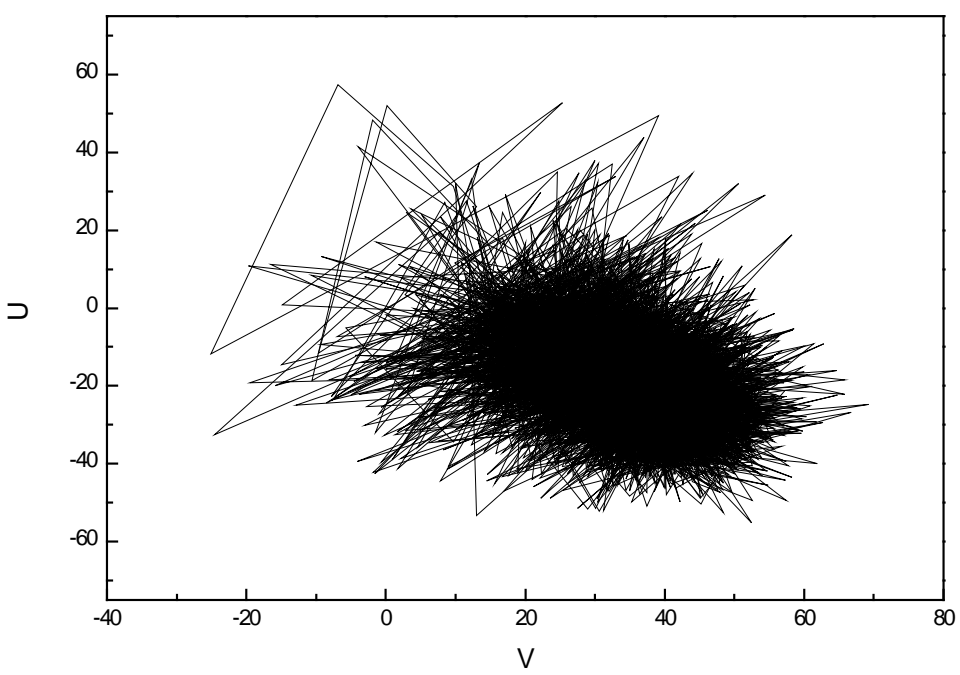

(d) $\mathrm{Ra}=9 \times 10^{5}$

Fig. 10 Phase space of velocity for (a) $R a=2 \times 10^{4}$, (b) $R a=4 \times 10^{4}$, (c) $R a=5 \times 10^{4}$, (d) $R a=9 \times 10^{5}$

\subsection{Unsteady flow characteristics}

In order to analyze nonlinear characteristics of the thermalsolutal convection with thermal and solutal source, two methods of recognizing route to chaos are provided, time evolution of concentration and the phase-space trajectory of velocity at the sample point $(18,15)$.

The effect of Rayleigh number on the route to chaos is illustrated for buoyance ratio $N c=-7.5$ in Fig. 9 and Fig. 10. It demonstrates from Fig. 9 (a) and Fig. 10 (a), the time history of solutes remains unchanged with time infinite long, and phase space is shrink into a limit point at $R a$ $=2 \times 10^{4}$, As Rayleigh number is increasing to $4 \times 10^{4}$, the limit point is lost and develops into periodic oscillations in the dimensionless time between 600 and 900, and the phase trajectory appears a limit cycle apparently as depicted by Fig. 9 (b) and Fig. 9 (b). It is found that there exist a critical Rayleigh number $R e_{c}$ of $3 \times 10^{4}$. If Rayleigh is lower than $R e_{c}$, flow state is stable. However, the flow loses its stability and turns into periodic oscillation at a Rayleigh number above $R a_{c}$. Further investigations for larger $\mathrm{Ra}$ are conducted, the oscillation system becomes complex evolving from periodic oscillation to a double periodic oscillation. It can be seen from the Fig. 9 (d) that periodic oscillations were broken and chaos occurs. Also, another critical one making the system from regular flow to chaos is obtained.

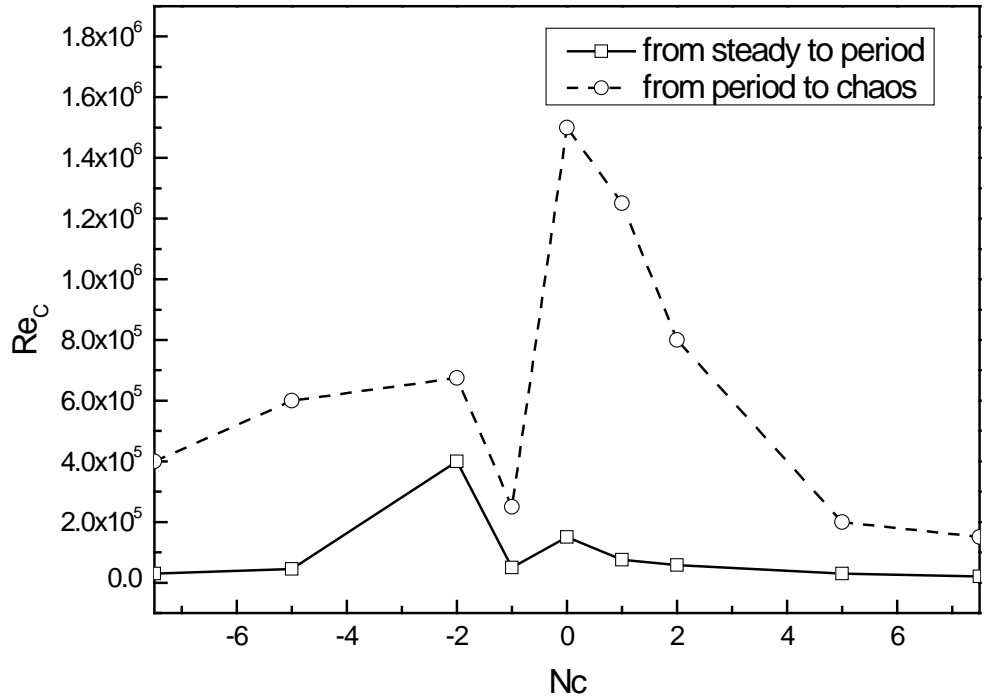

Fig. 11 Critical Rayleigh number with different $N C$ 
Figure 11 presents the effect of buoyancy ratio on the nonlinear system. The critical Rayleigh at which the convection changes from steady to periodic state and from periodic to chaotic state are summarized. It can be seen that, critical Rayleigh number is the lowest for $N c=-1$. Because when $N_{c}=-1$, the solutal buoyance is equal to thermal buoyancy but in opposite directions and a little $R a$ can destroy this balance. For the case of $N_{c}>-1$, the critical Rayleigh rises a lot, then declines and finally become flat. The reason is that power thermal buoyancy appears to destroy the steady and periodic state with increasing Nc. And stronger the solutal buoyance become, more unsteady system will be. Therefore critical one is decreasing further. For the case of $N_{c}<-1$, the trendy of $R a_{c}$ is consistent with the case of $N_{c}>-1$. It is also observed from the figure that the Rayleigh number from period to chaos is larger than that from steady to period state.

\section{CONCLUSION}

Numerical analysis of double diffusive convection with thermal and solutal source for different Rayleigh number, buoyance ratio, Soret and Dufour number have been carried out. The results show that asymmetric flow pattern will enhance the heat and mass transfer. Study result demonstrates that heat and mass transfer of thermosolutal convection are intense with increasing $N c$ and $R a$. It could be also concluded that the couple-diffusive interaction has linearly effect on heat and mass transfer. The results also show that double-diffusive develops from steady state, periodic oscillatory, a quasi-period oscillation to chaos. Additionally, flow pattern easily changes at lower critical Rayleigh number with the uprising buoyancy ratio.

\section{ACKNOWLEDGEMENTS}

This work is supported by Chinese National Natural Science Foundations under Grants 51736007 and 51476103, and China Scholarship Council.

\section{NOMENCLATURE}

C parametric concentration

$c, C_{\mathrm{h}}, C_{l}$ concentration, high concentration, low concentration $\left(\mathrm{kg} / \mathrm{m}^{3}\right)$

$D$ diffusion coefficient $\left(\mathrm{m}^{2} / \mathrm{s}\right)$

$D_{f} \quad$ Dufournumber

$g \quad$ gravity acceleration $\left(\mathrm{m} / \mathrm{s}^{2}\right)$,

$H \quad$ height of cavity (m)

Le Lewis number

$N c \quad$ buoyancy ratio

$\mathrm{Nu} \quad$ local Nusselt number

$\overline{\mathrm{Nu}} \quad$ average Nusselt number

$P \quad$ parametric pressure

$p \quad$ pressure $(\mathrm{Pa})$

$\mathrm{Pr} \quad$ Prandtl number

$\mathrm{Ra} \quad$ Rayleighnumber

$S_{r} \quad$ Soretnumber

Sh local Sherwood number

$\overline{S h} \quad$ average Sherwood number

$T, T_{\mathrm{h}}, T_{l} \quad$ temperature, high temperature, low temperature (K)

$t$ time (s)

$U \quad$ dimensionless velocity in $x$ direction

$u \quad$ velocity in $x$ direction $(\mathrm{m} / \mathrm{s})$

$V \quad$ dimensionless velocity in $x$ direction

$v \quad$ velocity in $y$ direction $(\mathrm{m} / \mathrm{s})$

$W \quad$ width of cavity (m)

$X \quad$ dimensionless $x$ coordinate

$Y \quad$ dimensionless $y$ coordinate

$x \quad x$ coordinate $(\mathrm{m})$

$y \quad y$ coordinate $(\mathrm{m})$

Greek Symbols $\alpha \quad$ thermal diffusion coefficient $\left(\mathrm{m}^{2} / \mathrm{s}\right)$

$\beta_{\mathrm{c}} \quad$ mass expansion coefficient $\left(\mathrm{m}^{3} / \mathrm{kg}\right)$

$\beta_{\mathrm{T}} \quad$ thermal expansion coefficient(1/K)

$\theta \quad$ dimensionless temperature

$v \quad$ Dynamic viscosity $\left(\mathrm{m}^{2} / \mathrm{s}\right)$

คo density, $\mathrm{kg} / \mathrm{m}^{3}$

$\tau \quad$ dimensionless time

$\kappa \mathrm{TC}, \kappa \mathrm{CT} \quad$ Dufour and Soret coefficient

Subscripts

l high value

$h \quad$ low value

\section{REFERENCES}

Altawallbeh, A.A., Bhadauria, B.S., and Hashim, I., 2013, "Linear and Nonlinear Double-diffusive Convection in a Saturated Anisotropic Porous Layer with Soret Effect and Internal Heat Source,” International Journal of Heat \& Mass Transfer, 59(1), 103-111. http://dx.doi.org/10.1016/j.ijheatmasstransfer.2012.12.005

Béghein, C., Haghighat, F., and Allard, F., 1992, "Numerical Study of Double-diffusive Natural Convection in a Square Cavity," International Journal of Heat \& Mass Transfer, 35(4), 833-846. http://dx.doi.org/10.1016/0017-9310(92)90251-m

Bhuvaneswari, M., Sivasankaran, S., and Kim, Y.J., 2011, "Numerical Study on Double Diffusive Mixed Convection with a Soret Effect in a Two-sided Lid-driven Cavity," Numerical Heat Transfer, Part A: Applications, 59(7): 543-560.

http://dx.doi.org/10.1080/10407782.2011.561077

Deepika, N., and Narayana, P.A.L., 2016, "Nonlinear Stability of Double-diffusive Convection in A Porous Layer with Throughflow and Concentration Based Internal Heat Source," Transport in Porous Media, 111(3), 751-762.

http://dx.doi.org/10.1007/s11242-016-0626-X

Gaikwad, S.N., Malashetty, M.S., and Prasad, K.R., 2007, “An Analytical Study of Linear and Non-linear Double Diffusive Convection with Soret and Dufour Effects in Couple Stress Fluid,” International Journal of Non-Linear Mechanics, 42(7), 903-913. http://dx.doi.org/10.1016/j.ijnonlinmec.2007.03.009

Gaikwad, S.N., and Kouser, S., 2014, "Double-diffusive Convection in a Couple Stress Fluid Saturated Porous Layer with Internal Heat Source,” International Journal of Heat \& Mass Transfer, 78(11), 1254-1264. http://dx.doi.org/10.1016/j.ijheatmasstransfer.2014.07.021

Hill, A.A., 2005, "Double-diffusive Convection in a Porous Medium with a Concentration Based Internal Heat Source," Proceedings Mathematical Physical \& Engineering Sciences, 461(2054), 561-574. http://dx.doi.org/10.2307/30046943

Juncu, G., 2005, “Conjugate-heat/mass Transfer from a Circular Cylinder with an Internal Heat/Mass Source in Laminar Crossflow at Low Reynolds Numbers," International Journal of Heat \& Mass Transfer, 48(2):419-424.

http://dx.doi.org/10.1016/j.ijheatmasstransfer.2004.09.020

Karimi, F., Xu, H.T., Wang Z.Y., Yang, M., and Zhang, Y.W., 2014, "Numerical Simulation of Unsteady Natural Convection from Heated Horizontal Circular Cylinders in a Square Enclosure," Numerical Heat Transfer, Part A: Applications, 65(8): 715-731. http://dx.doi.org/10.1080/10407782.2013.846607

Lin, J.H., Chen, C.K., Yang, and Zhang, Y.T., 2000, "The Inverse Estimation of the Thermal Boundary Behavior of a Heated Cylinder Normal to a Laminar Air Stream,” International Journal of Heat and Mass Transfer, 43 (21), 3991-4001.

http://dx.doi.org/10.1016/s0017-9310(00)00022-3 
Li, Z., Yang, M., Chen, Q.C., and Zhang, Y.W., 2013, “Numerical Solution of Melting in a Discretely Heated Enclosure Using an Interfacial Tracking Method," Numerical Heat Transfer, Part A: Applications, 64(11): 841-857.

http://dx.doi.org/10.1080/10407782.2013.807686

Malashetty, M.S., and Gaikwad, S.N., "Effect of Cross Diffusion on Double Diffusive Convection in the Presence of Horizontal Gradients," International Journal of Engineering Science, 2002, 40(7): 773-787. http://dx.doi.org/10.1016/s0020-7225(01)00097-0

Matta, A., and Hill, A.A., 2017, "Double-diffusive Convection in an Inclined Porous Layer with a Concentration-based Internal Heat Source,” Continuum Mechanics and Thermodynamics, 30(1), 165-173. http://dx.doi.org/10.1007/s00161-017-0592-8

Nazari, M., Louhghalam, L., and Kayhani, M.H., 2015, "Lattice Boltzmann Simulation of Double Diffusive Natural Convection in a Square Cavity with a Hot Square Obstacle," Chinese Journal of Chemical Engineering, 23(1), 22-30.

http://dx.doi.org/10.1016/j.cjche.2014.10.008

Nithyadevi, N., and Yang, R.J., 2009, "Double Diffusive Natural Convection in a Partially Heated Enclosure with Soret and Dufour Effects,” International Journal of Heat \& Fluid Flow, 30(5): 902-910. http://dx.doi.org/10.1016/j.ijheatfluidflow.2009.04.001

Platten, J.K., 2014, “The Soret Effect: A Review of Recent Experimental Results,” Journal of Applied Mechanics, 73(1), 5-15. http://dx.doi.org/10.1115/1.1992517

Sankar, M., Park, J., and Younghae, D.O., 2011, "Numerical Study of Natural Convection in a Vertical Porous Annulus with Internal Heat Source (ENG)," International Journal of Heat \& Mass Transfer, 54(7/8), 1493-1505. http://dx.doi.org/10.1016/j.ijheatmasstransfer.2010.11.043

Tasaka, Y., and Takeda, Y., 2005, "Effects of Heat Source Distribution on Natural Convection Induced by Internal Heating," International Journal of Heat \& Mass Transfer, 48(6), 1164-1174.

\section{http://dx.doi.org/10.1016/j.ijheatmasstransfer.2004.09.044}

Weaver, J.A., and Viskanta, R., 1991, "Natural Convection Due to Horizontal Temperature and Concentration Gradients-2. Species Interdiffusion, Soret and Dufour Effects," International Journal of Heat and Mass Transfer, 34, 3121e33.

http://dx.doi.org/10.1016/0017-9310(91)90080-X

Wang, J., Yang, M., and Zhang, Y.W., 2015, "Coupling-diffusive Effects on Thermosolutal Buoyancy Convection in a Horizontal Cavity," Numerical heat transfer, Part A, 68: 583-597. http://dx.doi.org/10.1080/10407782.2014.994412

Wang, J., Yang, M., and Zhang, Y.W., 2014, "Onset of Double-diffusive Convection in Horizontal Cavity with Soret and Dufour Effects," International Journal of Heat and Mass Transfer, 78, 1023-1031. http://dx.doi.org/10.1016/j.ijthermalsci.2016.03.012

Wang, J., Yang, M., He, Y.L., and Zhang, Y.W., 2016, “Oscillatory Double-diffusive Convection in a Horizontal Cavity with Soret and Dufour Effects,” International Journal of Thermal Sciences, 106(7): 5769.

http://dx.doi.org/10.1016/j.ijthermalsci.2016.03.012

Xu, H.T., Li, B.B., Chen, J., Luo, Q., Yan, Z.R., and Yang, M., 2016, "Lattice BGK Simulations of the Double Diffusive Mixed Convection in an Enclosure with Different Outlet Locations," International Journal of Heat and Mass Transfer, 92,158-172.

http://dx.doi.org/10.1016/j.ijheatmasstransfer.2015.08.100

Xu, H.T., Luo, Z.Q., Lou, Q., Zhang, S.Y., and Wang, J., 2019, “Lattice Boltzmann Simulations of the Double-diffusive Natural Convection and Oscillation,” International Journal of Thermal Sciences, 136, 159-171. http://dx.doi.org/10.1016/j.ijthermalsci.2018.10.015 